Laser Chem., 1999, Vol. 19, pp. 305-309

Reprints available directly from the publisher Photocopying permitted by license only
(C) 1999 OPA (Overseas Publishers Association) N.V.

Published by license under

the Harwood Academic Publishers imprint, part of The Gordon and Breach Publishing Group.

Printed in India.

\title{
INVESTIGATING THE ENVIRONMENT- DEPENDENT PHOTOPHYSICS OF CHLORINE DIOXIDE WITH RESONANCE RAMAN INTENSITIES
}

\author{
ANTHONY P. ESPOSITO, CATHERINE E. FOSTER \\ and PHILIP J. REID* \\ Dept. of Chemistry, University of Washington, Seattle, WA 98195, USA
}

(Received 7 April 1997)

\begin{abstract}
The condensed-phase excited-state reaction dynamics of chlorine dioxide are investigated using resonance Raman intensity analysis. Absolute Raman intensities are measured on resonance with the ${ }^{2} B_{1}-{ }^{2} A_{2}$ electronic transition and used to establish the excited-state structural evolution which occurs on the ${ }^{2} \mathrm{~A}_{2}$ surface following photoexcitation. Analysis of the intensities demonstrates that excited-state relaxation occurs along all three normal coordinates; however, only modest evolution is observed along the asymmetric stretch. This limited relaxation stands in contrast to the extensive motion along this coordinate in the gas phase. It is proposed that the initial excited-state structural relaxation serves to define the symmetry of the reaction coordinate and thus the mechanism of $\mathrm{Cl}$ production following photolysis of $\mathrm{OClO}$.
\end{abstract}

Keywords: Resonance Raman; chlorine dioxide; Raman intensities

\section{INTRODUCTION}

Understanding phase- and environment-dependent chemical reactivity is a central issue in environmental chemistry. This issue is nicely illustrated by the photochemistry of chlorine dioxide (OClO) (Fig. 1) [1]. Numerous investigations of $\mathrm{OClO}$ in the gas-phase have established that photoexcitation leads predominately to the formation

\footnotetext{
* Corresponding author.
} 


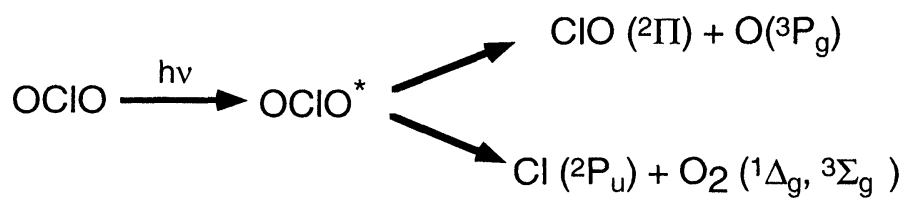

FIGURE 1 Schematic of OClO photochemistry. Photoexcitation of OClO can lead to either the formation of $\mathrm{ClO}$ and $\mathrm{O}$, or $\mathrm{Cl}+\mathrm{O}_{2}$.

of $\mathrm{ClO}$ and $\mathrm{O}$. However, in low-temperature matrices the situation is reversed with photoexcitation leading exclusively to the formation of $\mathrm{Cl}+\mathrm{O}_{2}$. The mechanistic reasons for this phase-dependent reactivity are unknown. To address this question, we have employed absolute resonance Raman intensity analysis to elucidate the excited-state reaction dynamics which occur following photolysis and the influence of solvent on these dynamics [2].

\section{EXPERIMENTAL AND RESULTS}

Resonance Raman spectra of $\mathrm{OClO}$ dissolved in cyclohexane were obtained throughout the photochemically relevant, ${ }^{2} \mathrm{~B}_{1}-{ }^{2} \mathrm{~A}_{2}$ electronic transition (Fig. 2). The direct and hydrogen shifted output of a $\mathrm{Nd}$ :YAG laser operating at $30 \mathrm{~Hz}$ was focused onto a flowing sample jet. Pulse energies were $\leq 10 \mu \mathrm{J}$ to minimize photolysis of OClO. The Raman scattering was collected utilizing a back-scattering geometry and delivered to a $0.5 \mathrm{~m}$ spectrograph equipped with a $\mathrm{LN}_{2}$ cooled $\mathrm{CCD}$ detector. Figure 3 presents the resonance Raman spectrum of OClO obtained with $368.9 \mathrm{~nm}$ excitation. Intensity in transitions involving all three normal coordinates (the symmetric stretch $\left(\nu_{1}\right)$, bend $\left(\nu_{2}\right)$, and asymmetric stretch $\left.\left(\nu_{3}\right)\right)$ is observed demonstrating that excited-state structural relaxation occurs along all three coordinates following photoexcitation. The electronic absorption spectrum and absolute resonance Raman intensities were modeled using the timedependent formalism of Raman scattering. The details of which have been presented elsewhere [2,3]. The excellent agreement between the predicted absorption and Raman cross-sections and the experimental results is shown in Figure 2. The picture of the excited state dynamics 


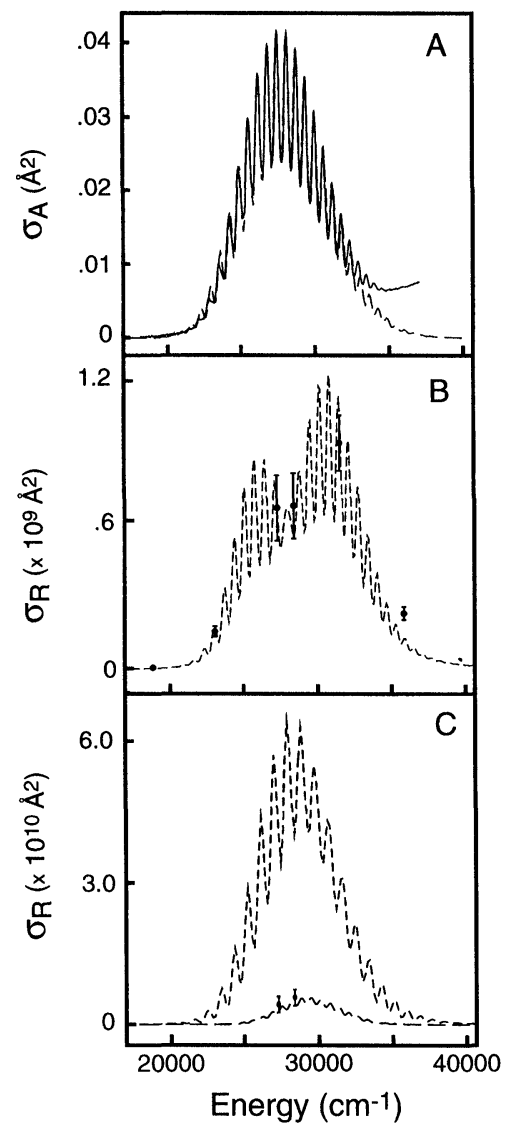

FIGURE 2 (A) Electronic absorption spectrum of $\mathrm{OClO}$ (solid) and fit determined by resonance Raman intensity analysis (dashed). Details of this analysis are presented elsewhere [2]. (B) Resonance Raman intensities of the symmetric stretch fundamental transition (points) and fit (dashed). (C) Resonance Raman intensities of the asymmetric stretch overtone transition (points) and predicted intensity using the parameters determined in this study (solid) as well as from analysis of the gas-phase absorption spectrum (dashed) [4].

derived from this analysis is one where the structural evolution is dominated by motion along the symmetric stretch with only limited evolution occurring along the bend and asymmetric stretch.

The modest evolution along the asymmetric stretch in solution is the most intriguing result to emerge from this study. The asymmetric 


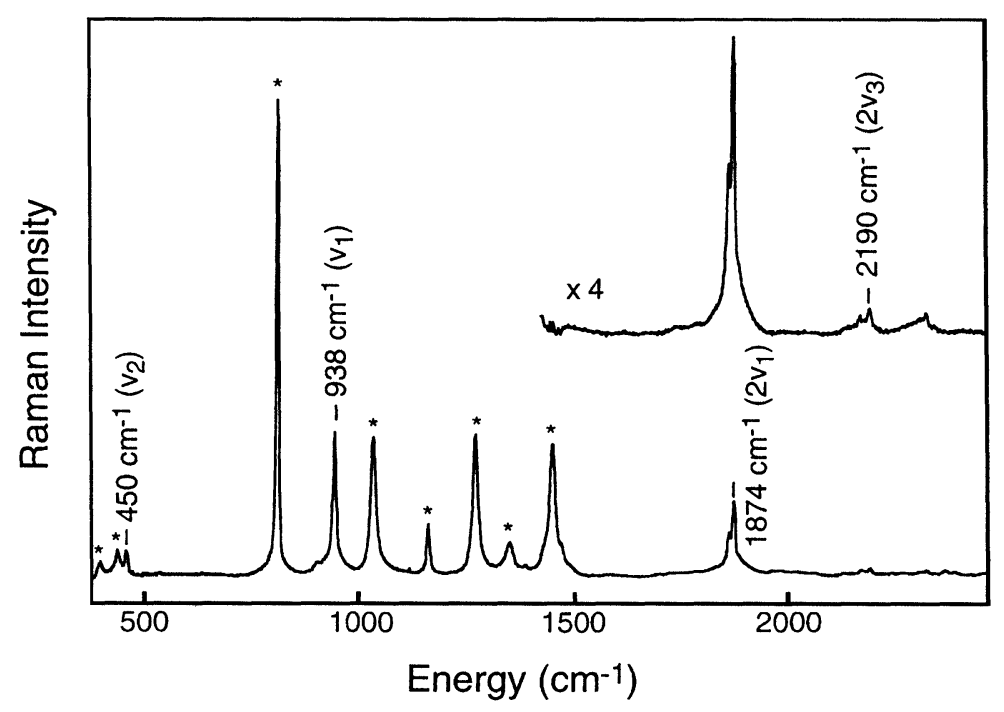

FIGURE 3 Resonance Raman spectrum of chlorine dioxide dissolved in cyclohexane obtained with $368.9 \mathrm{~nm}$ excitation. Sample concentration is $\sim 10 \mathrm{mM}$ and pulse energy is $10 \mu \mathrm{J} . \nu_{1}, \nu_{2}$, and $\nu_{3}$ correspond to the symmetric stretch, bend, and asymmetric stretch, respectively. Solvent scattering has been subtracted in the expanded view of the spectrum.

stretch is non-totally symmetric with respect to the $C_{2 v}$ symmetry of ground-state $\mathrm{OClO}$; therefore, fundamental intensity is not expected on resonance [3]. However, if the excited-state frequency differs from that of the ground state, then overtone intensity may be observed. Figure 1 demonstrates that overtone intensity assignable to the asymmetric stretch is observed. Modeling of this intensity suggests that this coordinate undergoes a modest reduction in excited-state frequency to $750 \mathrm{~cm}^{-1}$ from the $1100 \mathrm{~cm}^{-1}$ frequency of the ground state. This result stands in contrast to gas-phase absorption studies in which the excited-state potential surface along the asymmetric stretch was determined to have an energetic double minimum and a saddlepoint located at the Franck-Condon region [4]. Consistent with this, Figure $3 \mathrm{C}$ demonstrates that the gas-phase surface is not capable of reproducing the asymmetric stretch overtone intensity observed in cyclohexane. 


\section{DISCUSSION}

The results presented above suggest that the geometry established on the optically-prepared, ${ }^{2} \mathrm{~A}_{2}$ surface in cyclohexane is different than that in the gas phase. In particular, relaxation along the asymmetric stretch coordinate in the gas phase would result in a lowering of molecular symmetry from $\mathrm{C}_{2 \mathrm{v}}$ to $\mathrm{C}_{\mathrm{s}}$. However, the minimal evolution along this coordinate in cyclohexane results in preservation of the ground state symmetry. State correlation diagrams of $\mathrm{OClO}$ indicate that if $\mathrm{C}_{2 \mathrm{v}}$ symmetry is preserved, $\mathrm{Cl}+\mathrm{O}_{2}$ can be directly produced [1]. However, the reaction coordinate under $\mathrm{C}_{\mathrm{s}}$ symmetry should lead to the formation of $\mathrm{ClOO}$ which can subsequently decompose to form $\mathrm{Cl}+\mathrm{O}_{2}$. Therefore, the results presented here suggest that the dynamics which occur on the ${ }^{2} \mathrm{~A}_{2}$ surface may define the mechanism by which $\mathrm{Cl}$ is produced.

\section{Acknowledgements}

Acknowledgment is made to the Donors of The Petroleum Research Fund, administered by the American Chemical Society for support of this research. PJR is the recipient of a Camielle and Henry Dreyfus New Faculty Award.

\section{References}

[1] Vaida, V. and Simon, J. D. (1995). Science, 268, 1443.

[2] Esposito, A. P., Foster, C. E. and Reid, P. J., J. Phys. Chem., 101, 5309.

[3] Myers, A. B. and Mathies, R. A., Biological Applications of Raman Spectroscopy: Vol. 2 - Resonance Raman Spetra of Polyenes and Aromatics (John Wiley and Sons, Inc, New York, 1987) pp. 1-58.

[4] Richard, E. C. and Vaida, V. (1991). J. Chem. Phys., 94, 153. 